\title{
Entailment is Undecidable for Symbolic Heap Separation Logic Formulæ with Non-Established Inductive Rules
}

\author{
Mnacho Echenim ${ }^{\mathrm{a}}$, Radu Iosif ${ }^{\mathrm{b}}$, Nicolas Peltier ${ }^{\mathrm{a}}$ \\ ${ }^{a}$ Univ. Grenoble Alpes, CNRS, LIG, 700 Av. Centrale, 38000 Grenoble France \\ ${ }^{b}$ Univ. Grenoble Alpes, CNRS, VERIMAG, 700 Av. Centrale, 38000 Grenoble France
}

\begin{abstract}
Entailment is undecidable in general for Separation (SL) Logic formulæ with inductive definitions, but it has been shown to be decidable [1] if the inductive rules satisfy three conditions, namely progress, connectivity and establishment. We show that entailment is undecidable if the latter condition is dropped, thus drawing a much clearer frontier for (un)decidability.
\end{abstract}

Keywords: Separation Logic, Inductive Definition, Decidability

\section{Introduction}

Separation Logic (SL) is widely used in program verification to reason about programs manipulating dynamically allocated data structures [np]modif [2]. SL formulæ describe heaps, i.e. finite partial functions mapping locations (memory addresses) to tuples of locations (records) of a fixed size. [np]modif A location is allocated if it occurs in the domain of the considered heap. A formula $x \mapsto\left(y_{1}, \ldots, y_{\kappa}\right)$ states that the image of the location associated with $x$ in the heap is the tuple of locations associated with $\left(y_{1}, \ldots, y_{\kappa}\right)$, whereas $\phi_{1} * \phi_{2}$ states that the heap can be split into two disjoint parts satisfying $\phi_{1}$ and $\phi_{2}$, respectively. Describing unbounded data structures (lists, trees, etc.) is possible by the use of predicate symbols, the interpretation of which is given by a set of user-defined inductive rules. These SL formulæ, in which boolean conjunction does not occur and negation is restricted to disequalities, are commonly called symbolic heaps. Testing entailment between symbolic heaps is known to be decidable for a class of inductive definitions satisfying three natural conditions [1]:(1) progress, stating that every inductive rule allocates exactly one memory cell, (2) connectivity, 
ensuring that the locations allocated during the unfolding of a predicate form a tree-like connected structure and (3) establishment, stating that every existentially quantified variable introduced during the unfolding of inductive predicates must be eventually allocated (in the sense that it must be asserted equal to a variable $y$ such that an atom $y \mapsto(\ldots)$ occurs in the unfolding). These conditions play very different rôles. Condition (1) is actually not restrictive and is considered for simplicity only. In contrast, Condition (2) is crucial for decidability: entailments are undecidable for inductive definitions that are not connected, because then the inductive definitions may be used to encode context-free languages, the inclusion of which is known to be undecidable. The remaining question is whether condition (3) is actually required for decidability or whether it can be ignored. We show that discarding this condition leads to undecidability. The precise rôle of establishment for decidability of entailments between symbolic heaps has been an open problem since establishment was identified as a condition ensuring, with progress and connectivity, decidability of entailments between symbolic heaps [1]. The proof of [1] used a reduction of the entailment between symbolic heaps to the (decidable) satisfiability of monadic second order logic on graphs with bounded treewidth [3] and establishment was crucial to ensure that the considered heaps have a bounded treewidth. Later, a doubly exponential decision algorithm was designed [4], and we recently showed that establishment can be replaced by a strictly more general condition restricting the form of the disequations in the system [5]. The undecidability proof for non-established definitions given here implies the undecidability of entailments for definitions that cannot be transformed into the restricted disequations form [5].

\section{Separation Logic}

Let $\kappa \geq 0$ be a natural number, $\mathcal{V}$ and $\mathcal{C}$ be two disjoint sets of symbols (denoting respectively variables and constants ${ }^{1}$, and $\mathcal{P}$ be a set of predicate symbols. Each symbol $p \in \mathcal{P}$ is associated with a unique arity $\#(p) \geq 1$. The set of separation logic symbolic heaps $\phi$ is inductively defined as follows, where $x, x^{\prime}, y_{1}, \ldots, y_{\kappa}, z_{1}, \ldots, z_{\#(p)} \in \mathcal{V} \cup \mathcal{C}, u \in \mathcal{V}$ and $p \in \mathcal{P}$ :

$$
\phi::=x \approx x^{\prime}\left|x \not x^{\prime}\right| x \mapsto\left(y_{1}, \ldots, y_{\kappa}\right)|\phi * \phi| p\left(z_{1}, \ldots, z_{\#(p)}\right) \mid \exists u . \phi .
$$

\footnotetext{
${ }^{1}$ Constants are introduced only to improve readability. One could replace them by variables added as parameters to every predicate symbol, and adding disequality constraints $x \neq y$ for all $x, y \in \mathcal{C}$, stating that all constants are mapped to distinct locations. Hence the undecidability result holds also for $C=\emptyset$.
} 
A formula is predicate-free if it contains no symbols from $\mathcal{P}$. We denote by $\operatorname{var}(\phi)$ the set of variables freely occurring in $\phi$. A substitution is a partial mapping from $\mathcal{V}$ to $\mathcal{V} \cup \mathcal{C}$. The substitution of domain $\left\{x_{1}, \ldots, x_{n}\right\}$ mapping every variable $x_{i}$ to $t_{i}$ is denoted by $\left[x_{i} \leftarrow t_{i} \mid 1 \leq i \leq n\right]$. For every formula $\phi$ and substitution $\sigma$, $\phi \sigma$ denotes the formula obtained by replacing each free occurrence of a variable $x$ in $\phi$ by $\sigma(x)$ (bound variables are renamed to avoid collisions).

Let $\mathcal{U}$ be a countably infinite set of locations, with $\mathcal{C} \subseteq \mathcal{U}$. A structure is a pair $(\mathfrak{s}, \mathfrak{h})$ where $\mathfrak{s}$ is a store, i.e., a total mapping from $\mathcal{V} \cup \mathcal{C}$ to $\mathcal{U}$ that is the identity on $\mathcal{C}$; and $\mathfrak{h}$ is a heap, i.e., a partial finite mapping from $\mathcal{U}$ to $\mathcal{U}^{\mathrm{K}}$. Two heaps $\mathfrak{h}_{1}$ and $\mathfrak{h}_{2}$ are disjoint iff $\operatorname{dom}\left(\mathfrak{h}_{1}\right) \cap \operatorname{dom}\left(\mathfrak{h}_{2}\right)=\emptyset$, and $\mathfrak{h}_{1} \uplus \mathfrak{h}_{2}$ denotes the union of disjoint heaps.

A system of inductive definitions (SID) $\mathcal{R}$ is a set of rules $p\left(x_{1}, \ldots, x_{\#(p)}\right) \Leftarrow$ $\phi$, where $x_{1}, \ldots, x_{\#(p)}$ are pairwise distinct variables and $\phi$ is a symbolic heap with $\operatorname{var}(\phi) \subseteq\left\{x_{1}, \ldots, x_{\#(p)}\right\}$. A system $\mathcal{R}$ is progressing and connected if every rule in $\mathcal{R}$ is of the form $p\left(x_{1}, \ldots, x_{\#(p)}\right) \Leftarrow \exists y_{1}, \ldots, y_{n} . x_{1} \mapsto\left(u_{1}, \ldots, u_{\#(p)}\right) *$ $*_{i=1}^{m} p_{i}\left(y_{1}^{i}, \ldots, y_{\#\left(p_{i}\right)}^{i}\right)$, where for all $i \in \llbracket 1, m \rrbracket$, we have $y_{1}^{i} \in\left\{u_{1}, \ldots, u_{\#(p)}\right\}$.

For any formula $\phi$, we write $\phi \rightarrow_{\mathcal{R}} \psi$ if $\psi$ is obtained from $\phi$ by replacing an occurrence of a predicate atom $p\left(x_{1}, \ldots, x_{\#(p)}\right)$ by $\rho\left[y_{i} \leftarrow x_{i} \mid 1 \leq i \leq \#(p)\right]$, where $\mathcal{R}$ contains a rule $p\left(y_{1}, \ldots, y_{\#(p)}\right) \Leftarrow \rho$ and the existential variables of $\rho$ are $\alpha$-renamed to avoid collisions. As usual, $\rightarrow_{\mathcal{R}}^{*}$ denotes the reflexive and transitive closure of $\rightarrow_{\mathcal{R}}$. A formula $\psi$ such that $\phi \rightarrow_{\mathcal{R}}^{*} \psi$ is called an $\mathcal{R}$-unfolding of $\phi$. A SID $\mathcal{R}$ is established if, in every predicate-free $\mathcal{R}$-unfolding $\psi$ of a predicate atom $p\left(x_{1}, \ldots, x_{\#(p)}\right)$, each existentially quantified variable in $\psi$ is asserted equal to a variable $x$, where $x \mapsto\left(y_{1}, \ldots, y_{\kappa}\right)$ is a an atom of $\psi$.

For all structures $(\mathfrak{s}, \mathfrak{h})$, symbolic heaps $\phi$ containing no predicate atom and SIDs $\mathcal{R}$, we write $(\mathfrak{s}, \mathfrak{h})=\phi$ iff one of the following holds: (i) $\phi \equiv x \approx x^{\prime}$ (resp. $\left.\phi \equiv x \not \approx x^{\prime}\right), \operatorname{dom}(\mathfrak{h})=\emptyset$ and $\mathfrak{s}(x)=\mathfrak{s}\left(x^{\prime}\right)$ (resp. $\mathfrak{s}(x) \neq \mathfrak{s}\left(x^{\prime}\right)$ ); (ii) $\phi \equiv x \mapsto$ $\left(y_{1}, \ldots, y_{\kappa}\right), \operatorname{dom}(\mathfrak{h})=\{\mathfrak{s}(x)\}$ and $\mathfrak{h}(\mathfrak{s}(x))=\left(\mathfrak{s}\left(y_{1}\right), \ldots, \mathfrak{s}\left(y_{\kappa}\right)\right) ; \quad$ (iii) $\phi \equiv \phi_{1} * \phi_{2}$ and $\mathfrak{h}=\mathfrak{h}_{1} \uplus \mathfrak{h}_{2}$ where $\left(\mathfrak{s}, \mathfrak{h}_{i}\right) \models \phi_{i}$, for all $i \in\{1,2\}$; (iv) $\phi \equiv \exists x$. $\phi^{\prime}$ and there exists a store $\mathfrak{s}^{\prime}$ coinciding with $\mathfrak{s}$ on all variables and constants distinct from $x$ such that $\left(\mathfrak{s}^{\prime}, \mathfrak{h}\right) \models \phi^{\prime}$. This definition is extended to formulæ containing predicate symbols as follows: $(\mathfrak{s}, \mathfrak{h}) \models_{\mathcal{R}} \phi$ iff $(\mathfrak{s}, \mathfrak{h}) \models_{\mathcal{R}} \psi$, for some predicate-free $\mathcal{R}$-unfolding $\psi$ of $\phi$. [np]modifs Note that (dis)equations are satisfied only in empty heaps. This slightly departs from usual definitions $[1,2]$ but simplifies notations by getting rid of standard conjunctions. 


\section{Encoding the Post Correspondence Problem}

The undecidability proof uses a reduction from a variant of Post's Correspondence Problem (PCP). We denote by $w[i]$ the $i$-th character of the word $w$, and by $|w|$ its length, so that $w=(w[1], \ldots, w[|w|])$. We recall that the PCP asks, given two sequences $\mathbf{u}^{i}=\left(\mathbf{u}_{1}^{i}, \ldots, \mathbf{u}_{N}^{i}\right)$ (with $\left.i=1,2\right)$ of words on the same alphabet $\mathbb{V}$, whether there exists a nonempty sequence $\mathbf{s}=\left(\mathbf{s}_{1}, \ldots, \mathbf{s}_{G}\right)$ of elements of $\llbracket 1, N \rrbracket$ (called a solution of the PCP) such that $\mathbf{u}_{\mathbf{s}_{1}}^{1} \ldots . \mathbf{u}_{\mathbf{s}_{G}}^{1}=\mathbf{u}_{\mathbf{s}_{1}}^{2} \ldots \ldots \mathbf{u}_{\mathbf{s}_{G}}^{2}$. The latter word is called a witness of the PCP and its length $\Sigma_{g=1}^{G}\left|\mathbf{u}_{g}^{i}\right|$ will be denoted by $L$. PCP is a well-known undecidable problem. We assume that all the words in $\mathbf{u}^{i}$ are nonempty (which does not impact the undecidability proof of the PCP). We consider a slightly adapted version of the PCP, in which: (i) The last element of both sequences of words is a special character $\$=\mathbf{u}_{N}^{1}=\mathbf{u}_{N}^{2}$, not occurring in the words $\mathbf{u}_{n}^{i}$, for $i \in\{1,2\}, n \in \llbracket 1, N-1 \rrbracket$; and (ii) any solution must be such that $G>1$, $\mathbf{s}_{G}=N$ and $\mathbf{s}_{g} \neq N$ if $g<G$. It is clear that the standard PCP can be reduced to the adapted version, which is thus also undecidable. We denote by $M^{i}$ the maximal length of the words in $\mathbf{u}^{i}$, i.e. $M^{i} \stackrel{\text { def }}{=} \max \left\{\left|\mathbf{u}_{n}^{i}\right| \mid n \in \llbracket 1, N \rrbracket\right\}$, for $i \in\{1,2\}$ and let $M \stackrel{\text { def }}{=} \max \left(\left\{M_{1}, M_{2}\right\}\right)$.

We consider two special constants nil and $\perp$, and we associate all natural numbers $m \in \llbracket 1, M \rrbracket$ and all elements $a \in \mathbb{V}$ with pairwise distinct constants also distinct from nil and $\perp$. These constants will be, for the sake of readability, also denoted by $m$ or $a$ (assuming, w.l.o.g., that $\mathbb{V} \cup \mathbb{N} \cup\{\perp$, nil $\} \subseteq C$ and $\mathbb{V} \cap \mathbb{N}=\emptyset$ ). We assume next that $\kappa=6$, i.e. we fix the number of record fields. The encoding could easily be adapted for any $\kappa \geq 2$, using binary trees to represent tuples of elements, but this would greatly hinder readability, because of the progress condition which requires that every rule allocates exactly one location. For readability, we shall actually consider elements referring to tuples of any length $n \leq 6$, where missing elements are implicitly replaced by the constant $\perp$. Hence the notation $x \mapsto\left(y_{1}, \ldots, y_{n}\right)$ for $n \leq 6$ stands for $x \mapsto\left(y_{1}, \ldots, y_{n}, \perp^{6-n}\right)$. For the sake of conciseness, we allow disjunctions in the right-hand side of the inductive rules: a rule $p(\vec{x}) \Leftarrow \phi *\left(\psi_{1} \vee \psi_{2}\right)$ stands for $\left\{p(\vec{x}) \Leftarrow \phi * \psi_{1}, p(\vec{x}) \Leftarrow \phi * \psi_{2}\right\}$. We first show that the solutions of the PCP can be encoded by structures of a specific form.

Definition 1. A heap $\mathfrak{h}$ is a PCP-encoding if there exists a word $w$ with $L \stackrel{\text { def }}{=}$ $|w|>1$, a natural number $G>1$ and pairwise distinct locations $\ell_{1}, \ldots, \ell_{L+1}$ and $\ell_{1}^{\prime}, \ldots, \ell_{G+1}^{\prime}$ with $\ell_{L+1}=\ell_{G+1}^{\prime}=$ nil, such that:

1. $\operatorname{dom}(\mathfrak{h})=\left\{\ell_{1}, \ldots, \ell_{L}, \ell_{1}^{\prime}, \ldots, \ell_{G}^{\prime}\right\}$. 
2. For every $l \in \llbracket 1, L \rrbracket, \mathfrak{h}\left(\ell_{l}\right)$ is of the form $\left(\ell_{l+1}, w[l], \ell_{l}^{1}, p_{l}^{1}, \ell_{l}^{2}, p_{l}^{2}\right)$, for locations $\ell_{l}^{1}, \ell_{l}^{2}$ and integers $p_{l}^{1}, p_{l}^{2} \in \llbracket 1, M \rrbracket$, with $\ell_{1}^{1}=\ell_{1}^{2}=\ell_{1}^{\prime}, \ell_{L}^{1}=\ell_{L}^{2}=\ell_{G}^{\prime}$ and $p_{1}^{1}=p_{1}^{2}=p_{L}^{1}=p_{L}^{2}=1$.

3. For every $g \in \llbracket 1, G \rrbracket, \mathfrak{h}\left(\ell_{g}^{\prime}\right)$ is of the form $\left(\ell_{g+1}^{\prime}, \mathbf{s}_{g}\right)$ for some $\mathbf{s}_{g} \in \llbracket 1, N \rrbracket$, with $\mathbf{s}_{G}=N$ and $\mathbf{s}_{g} \neq N$ if $g<G$.

The location $\ell_{1}$ is called the root of $\mathfrak{h}$. We denote $w(\mathfrak{h}) \stackrel{\text { def }}{=} w$ and $\mathbf{s}(\mathfrak{h}) \stackrel{\text { def }}{=}$ $\left(\mathbf{s}_{1}, \ldots, \mathbf{s}_{G}\right)$; clearly $\mathfrak{h}$ uniquely defines $w(\mathfrak{h})$ and $\mathbf{s}(\mathfrak{h})$.

[np]capital h, for consistency in titles?

The sequence $\ell_{1}, \ldots, \ell_{L}$ encodes the sequence of characters $w[1], \ldots$, $w[L]$ in a witness $w$. Each location $\ell_{l}$ refers to a tuple containing the next element of the sequence $\ell_{l+1}$ as well as the character $w[l]$, and locations $\ell_{l}^{1}, p_{l}^{1}, \ell_{l}^{2}, p_{l}^{2}$, which will be used to

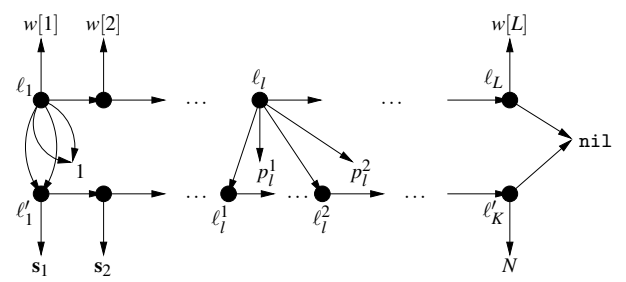

Figure 1: Encoding of a PCP Solution by a Heap denote the position of the character within the words $\mathbf{u}_{\mathbf{s}_{1}}^{1} \ldots . \mathbf{u}_{\mathbf{s}_{G}}^{1}$ and $\mathbf{u}_{\mathbf{s}_{1}}^{2} \ldots . \mathbf{u}_{\mathbf{s}_{G}}^{2}$ (see Def. 2 ). The sequence $\ell_{1}^{\prime}, \ldots, \ell_{G}^{\prime}$ represents the solution s. Each location $\ell_{g}^{\prime}$ points to a tuple containing the next element of the sequence $\ell_{g+1}^{\prime}$ and the $g$-th component of $\mathbf{s}$ (see Fig. 1).

Definition 2. A PCP-encoding $\mathfrak{h}$ is $i$-consistent for $i=1,2$ if, for all $l \in \llbracket 1, L \rrbracket$, there exists $g \in \llbracket 1, G \rrbracket$ such that, with the notations of Def. $1, \ell_{l}^{i}=\ell_{g}^{\prime}$ and:

1. $w[l]$ is the $p_{l}^{i}$-th character of the word $\mathbf{u}_{\mathbf{s}_{g}}^{i}$ (i.e., $\left.w[l]=\mathbf{u}_{\mathbf{s}_{g}}^{i}\left[p_{l}^{i}\right]\right)$.

2. If $l \neq L$ and $p_{l}^{i}<\left|\mathbf{u}_{\mathbf{s}_{g}}^{i}\right|$ then $\ell_{l+1}^{i}=\ell_{g}^{\prime}$ and $p_{l+1}^{i}=p_{l}^{i}+1$.

3. If $l \neq L$ and $p_{l}^{i}=\left|\mathbf{u}_{\mathbf{s}_{g}}^{i}\right|$ then $g<G, \ell_{l+1}^{i}=\ell_{g+1}^{\prime}$ and $p_{l+1}^{i}=1$.

Lemma 3. A PCP admits a solution iff there exists a PCP-encoding that is both 1-consistent and 2-consistent.

Proof. Assume that the PCP admits a solution s of length $G$, with witness $w$ and let $L=|w|$. We construct a PCP-encoding as follows. Let $\ell_{1}, \ldots, \ell_{L}, \ell_{1}^{\prime}, \ldots, \ell_{G}^{\prime}$ be pairwise distinct locations distinct from all constants. Let $\ell_{L+1} \stackrel{\text { def }}{=} \ell_{G^{\prime}+1}^{\prime} \stackrel{\text { def }}{=}$ nil, and let $\mathfrak{h}$ be a heap of domain $\left\{\ell_{1}, \ldots, \ell_{L}, \ell_{1}^{\prime}, \ldots, \ell_{G}^{\prime}\right\}$, such that: (i) For every $l \in \llbracket 1, L \rrbracket, \mathfrak{h}\left(\ell_{l}\right) \stackrel{\text { def }}{=}\left(\ell_{l+1}, w[l], \ell_{g_{l}^{1}}^{\prime}, p_{l}^{1}, \ell_{g_{l}^{2}}^{\prime}, p_{l}^{2}\right)$, where $g_{l}^{i}$ is the greatest element in $\llbracket 1, G \rrbracket$ with $\left|\mathbf{u}_{\mathbf{s}_{1}}^{i} \ldots . . \mathbf{u}_{\mathbf{s}_{g_{l}-1}}^{i}\right|<l$, and $p_{l}^{i} \stackrel{\text { def }}{=} l-\left|\mathbf{u}_{\mathbf{s}_{1}}^{i} \ldots . \mathbf{u}_{\mathbf{s}_{g_{l}-1}}^{i}\right| . \quad$ (ii) For every 
$g \in \llbracket 1, G \rrbracket, \mathfrak{h}\left(\ell_{g}^{\prime}\right) \stackrel{\text { def }}{=}\left(\ell_{g+1}^{\prime}, \mathbf{s}_{g}\right)$. It is straightforward to check that $\mathfrak{h}$ is a PCPencoding. Conversely, let $\mathfrak{h}$ be a PCP-encoding that is $i$-consistent for all $i=1,2$, and let $w \stackrel{\text { def }}{=} w(\mathfrak{h})$ and $\mathbf{s} \stackrel{\text { def }}{=} \mathbf{s}(\mathfrak{h})$. By induction on $l$, we can show, by a case analysis on whether $p_{l}^{i}<\left|\mathbf{u}_{\mathbf{s}_{l}^{i}}^{i}\right|$ or not, and using Conditions 2 and 3 of Def.

2 that [np]change here, because there was a latex pb with command either for every $i \in\{1,2\}$ and $l \in \llbracket 1, L \rrbracket$, the following equality holds: $w[1] \ldots w[l]=$ $\mathbf{u}_{\mathbf{s}_{1}}^{i} \ldots . \mathbf{u}_{\mathbf{s}_{g_{l}-1}^{i}}^{i} \cdot \mathbf{u}_{\mathbf{s}_{g_{l}}^{i}}^{i}[1] \ldots . \mathbf{u}_{\mathbf{s}_{g_{l}}}^{i}\left[p_{l}^{i}\right](1)$.

$$
\begin{aligned}
& I(x) \Leftarrow \exists x^{\prime}, y, z \cdot x \mapsto\left(x^{\prime}, \mathrm{a}, y, 1, y, 1\right) * W\left(x^{\prime}, z\right) * S(y, z) \\
& W(x, z) \Leftarrow \exists x^{\prime}, y_{1}, y_{2} . x \mapsto\left(x^{\prime}, \mathrm{a}, y_{1}, \mathrm{p}_{1}, y_{2}, \mathrm{p}_{2}\right) * W\left(x^{\prime}, z\right) \\
& W(x, z) \Leftarrow x \mapsto(\mathrm{nil}, \$, z, 1, z, 1) \\
& S(y, z) \Leftarrow \exists y^{\prime} \cdot y \mapsto\left(y^{\prime}, \mathrm{n}\right) * S\left(y^{\prime}, z\right) \quad \text { if } \mathrm{n}<N \\
& S(y, z) \Leftarrow y \mapsto(\operatorname{nil}, N) * y \approx z \\
& A(x) \Leftarrow \exists x^{\prime}, a, z_{1}, p_{1}, z_{2}, p_{2} . x \mapsto\left(x^{\prime}, a, z_{1}, p_{1}, z_{2}, p_{2}\right) * A\left(x^{\prime}\right) \\
& A(x) \Leftarrow \exists x^{\prime}, a, z_{1}, p_{1}, z_{2}, p_{2} . x \mapsto\left(x^{\prime}, a, z_{1}, p_{1}, z_{2}, p_{2}\right) \\
& C\left(x, x^{\prime}, a, z_{1}, p_{1}, z_{2}, p_{2}\right) \Leftarrow x \mapsto\left(x^{\prime}, a, z_{1}, p_{1}, z_{2}, p_{2}\right) * A\left(x^{\prime}\right) \\
& C\left(x, x^{\prime}, a, z_{1}, p_{1}, z_{2}, p_{2}\right) \Leftarrow x \mapsto\left(x^{\prime}, a, z_{1}, p_{1}, z_{2}, p_{2}\right) \\
& B(x) \Leftarrow \exists x^{\prime}, a, y \cdot x \mapsto\left(x^{\prime}, a, y, 1, y, 1\right) * B\left(x^{\prime}\right) * A(y) \\
& B(x) \Leftarrow \exists x^{\prime}, a, z_{1}, p_{1}, z_{2}, p_{2} . x \mapsto\left(x^{\prime}, a, z_{1}, p_{1}, z_{2}, p_{2}\right) * B\left(x^{\prime}\right) \\
& B(x) \Leftarrow \exists x^{\prime}, a, z_{1}, z_{2}, p_{2}, z_{1}^{\prime} \cdot x \mapsto\left(x^{\prime}, a, z_{1}, \mathrm{p}_{1}, z_{2}, p_{2}\right) * A\left(x^{\prime}\right) \\
& * C\left(z_{1}, z_{1}^{\prime}, \mathrm{n}, \perp, \perp, \perp, \perp\right) * a \neq \mathbf{u}_{n}^{1}\left[p_{1}\right] \text { if } p_{1} \leq\left|\mathbf{u}_{\mathrm{n}}^{1}\right| \\
& B(x) \Leftarrow \exists x^{\prime}, a, z_{1}, z_{2}, p_{2}, a^{\prime}, z_{1}^{\prime}, p_{1}^{\prime}, z_{2}^{\prime}, p_{2}^{\prime}, z_{1}^{\prime \prime} \cdot x \mapsto\left(x^{\prime}, a, z_{1}, \mathrm{p}_{1}, z_{2}, p_{2}\right) \\
& * C\left(x^{\prime}, a^{\prime}, z_{1}^{\prime}, p_{1}^{\prime}, z_{2}^{\prime}, p_{2}^{\prime}\right) * C\left(z_{1}, z_{1}^{\prime \prime}, \mathrm{n}, \perp, \perp, \perp, \perp\right) \\
& *\left(z_{1}^{\prime} \not \approx z_{1} \vee p_{1}^{\prime} \not \approx p_{1}+1\right) \text { if } p_{1}<\left|\mathbf{u}_{\mathrm{n}}^{1}\right| \\
& B(x) \Leftarrow \exists x^{\prime}, a, z_{1}, z_{2}, p_{1}^{\prime}, p_{2}, a^{\prime}, z_{1}^{\prime}, z_{2}^{\prime}, p_{2}^{\prime}, z_{1}^{\prime \prime} . x \mapsto\left(x^{\prime}, a, z_{1}, \mathrm{p}_{1}, z_{2}, p_{2}\right) \\
& * C\left(x^{\prime}, a^{\prime}, z_{1}^{\prime}, p_{1}^{\prime}, z_{2}^{\prime}, p_{2}^{\prime}\right) * C\left(z_{1}, z_{1}^{\prime \prime}, \mathrm{n}, \perp, \perp, \perp, \perp\right) \\
& *\left(p_{1}^{\prime} \not \approx \vee z_{1}^{\prime} \not z_{1}^{\prime \prime}\right) \text { if } p_{1}=\left|\mathbf{u}_{\mathrm{n}}^{1}\right|
\end{aligned}
$$

Figure 2: The SID $\mathcal{R}^{\pi}$ for the PCP Instance $\pi=\left(\mathbf{u}^{1}, \mathbf{u}^{2}\right)$ (for all $\mathrm{a} \in \mathbb{V}, \mathrm{p}_{1}, \mathrm{p}_{2} \in \llbracket 1, M \rrbracket, \mathrm{n} \in \llbracket 1, N \rrbracket$ )

Let $\pi=\left(\mathbf{u}^{1}, \mathbf{u}^{2}\right)$ be an instance of PCP, where $\mathbf{u}^{i}=\left(\mathbf{u}_{1}^{i}, \ldots, \mathbf{u}_{N}^{i}\right), i=1,2$. We show how to transform $i$-consistent PCP-encodings into SL formulæ. Let $\mathcal{R}^{\pi}$ be the set of rules in Fig. 2. We show that $I$ defines the PCP-encodings of $\pi$ (Def. 1). [np]modifs Intuitively $W$ and $S$ allocate the locations $\ell_{1}, \ldots, \ell_{L+1}$ and 
$\ell_{1}^{\prime}, \ldots, \ell_{G+1}^{\prime}$ respectively. The existential variables $y_{1}, y_{2}$ in (3) are not allocated (the corresponding pointers $w[1], w[2], \ldots$ in Fig. 1 are pending), hence this rule is not established.

Lemma 4. $(\mathfrak{s}, \mathfrak{h}) \models_{\mathcal{R}^{\pi}} I\left(x_{1}\right)$ iff $\mathfrak{h}$ is a PCP-encoding of root $\mathfrak{s}\left(x_{1}\right)$.

Proof. $(\mathfrak{s}, \mathfrak{h}) \models_{\mathcal{R}^{\pi}} I\left(x_{1}\right)$ iff $(\mathfrak{s}, \mathfrak{h}) \models \phi$ for some predicate-free formula $\phi$ with $I\left(x_{1}\right) \rightarrow_{\mathcal{R}^{\pi}}^{*} \phi$. By Rule (2), I( $\left.x_{1}\right) \rightarrow_{\mathcal{R}^{\pi}}^{*} \phi$ iff there exists $a_{1} \in \mathbb{V}$ s.t. $\exists x_{2}, y_{1}, z . x_{1} \mapsto$ $\left(x_{2}, a_{1}, y_{1}, 1, y_{1}, 1\right) * W\left(x_{2}, z\right) * S\left(y_{1}, z\right) \rightarrow_{R^{\pi}}^{*} \phi$. Any $\rightarrow_{\mathcal{R}^{\pi} \text {-derivation from } W\left(x_{2}, z\right)}$ starts by a sequence of application of Rule (3) followed by one application of (4). Thus $I\left(x_{1}\right) \rightarrow_{\mathcal{R}^{\pi}}^{*} \phi$ iff there exists $L \in \mathbb{N}, a_{1}, \ldots, a_{L-1} \in \mathbb{V}, p_{2}^{1}, p_{2}^{2}, \ldots, p_{L-1}^{1}, p_{L-1}^{2} \in$ $\llbracket 1, M \rrbracket$ s.t. $\exists x_{2}, \ldots, x_{L}, y_{1}, z . x_{1} \mapsto\left(x_{2}, a_{1}, y_{1}, 1, y_{1}, 1\right) * \psi * S\left(y_{1}, z\right) \rightarrow_{\mathcal{R}^{\pi}}^{*} \phi$, where $\psi \stackrel{\text { def }}{=} *_{l=2}^{L-1} x_{l} \mapsto\left(x_{l+1}, a_{l}, y_{l}^{1}, p_{l}^{1}, y_{l}^{2}, p_{l}^{2}\right) * x_{L} \mapsto(\mathrm{nil}, \$, z, 1, z, 1)$. Applying Rule (5) $G-1$ times and then Rule (6), we deduce that $I\left(x_{1}\right) \rightarrow_{\mathcal{R}^{\pi}}^{*} \phi$ iff there exists $L \in \mathbb{N}, G \in \mathbb{N}, a_{1}, \ldots, a_{L-1} \in \mathbb{V}, p_{2}^{1}, p_{2}^{2}, \ldots, p_{L-1}^{1}, p_{L-1}^{2} \in \llbracket 1, M \rrbracket, n_{1}, \ldots, n_{G-1} \in$ $\llbracket 1, N-1 \rrbracket$ s.t. $\exists x_{2}, \ldots, x_{L}, y_{1}, \ldots, y_{G} . x_{1} \mapsto\left(y, a_{1}, y_{1}, 1, y_{1}, 1\right) * \psi * \psi^{\prime} \rightarrow_{\mathcal{R}}^{*} \phi$ where $\psi^{\prime} \stackrel{\text { def }}{=} *_{g=1}^{G-1} y_{g} \mapsto\left(y_{g+1}, n_{g}\right) * y_{G} \mapsto(\mathrm{nil}, N) * y_{G} \approx z$. Using this equivalence, it is easy to check that $(\mathfrak{s}, \mathfrak{h}) \models I\left(x_{1}\right)$ iff $\mathfrak{h}$ is a PCP-encoding with root $\mathfrak{s}\left(x_{1}\right)$.

Predicate $B$ defines the PCP-encodings that are not $i$-consistent, for some $i=1,2$ (Def. 2). Such structures necessarily contain locations $\ell_{l}$ and $\ell_{g}^{\prime}$ contradicting one of Cond. 1, 2 or 3 from Def. 2. Rule (11) is applied when $\ell_{g}^{\prime}$ is such that $g>1$. It allocates the first element $\ell_{1}$ of the witness, then invokes $A(y)$ to allocate all elements $\ell_{1}^{\prime}, \ldots, \ell_{g-1}^{\prime}$, and the predicate $B\left(x^{\prime}\right)$ to allocate the remaining elements. Note that, [np]modifs thanks to the rules (7) and (8), $A$ allocates arbitrary linked structures, with the link on the first record field (all the pointers in the list are pending and the resulting list does not necessarily end with nil). Predicate $C$ is similar to $A$, but has 6 additional parameters denoting the heap image of the first allocated location. Rule (12) is applied $l-2$ times to allocate locations $\ell_{2}, \ldots, \ell_{l-1}$ and also to allocate $\ell_{1}$ in the case where $g=1$ (this case is considered apart since the sequence $\ell_{1}^{\prime}, \ldots, \ell_{g-1}^{\prime}$ is empty). The remaining rules allocate the faulty location $\ell_{l}$. Rules (13-15) handle the case where the heap is not 1-consistent. Rule (13) allocates a location contradicting Cond. 1 in Def. 2. The predicates $A(y)$ and $C\left(z_{1}, y^{\prime}, n, \perp, \perp, \perp, \perp\right)$ are used to allocate locations $\ell_{l+1}, \ldots, \ell_{L}$, and $\ell_{g}^{\prime}, \ldots, \ell_{G}^{\prime}$, respectively. Similarly, Rules (14) and (15) allocate a location contradicting Cond. 2 or 3 in Def. 2, respectively. The rules corresponding to the case where the heap is 2-consistent are defined in a similar way (they are omitted for conciseness). 
Lemma 5. Let $\mathfrak{h}$ be a PCP-encoding and let $\mathfrak{s}$ be a store such that $\mathfrak{s}(x)$ is the root of $\mathfrak{h}$. The following equivalence statement holds: $(\mathfrak{s}, \mathfrak{h}) \models_{\mathfrak{R}} B(x)$ iff $\mathfrak{h}$ is not $i$-consistent, for some $i=1,2$.

Proof. We use the same notations as in Def. 1. $\Rightarrow$ : Assume that $(\mathfrak{s}, \mathfrak{h}) \models_{\mathcal{R}^{\pi}} B(x)$. Then there exists a predicate-free unfolding $\phi$ of $B(x)$ such that $(\mathfrak{s}, \mathfrak{h}) \models \mathcal{R}^{\pi} \phi$. By definition of the rules defining $B$, this entails that one of rules (13), (14) or (15) (or the corresponding rules for $i=2$ ) must have been applied at some point in the derivation, since these are the only rules that can eliminate the predicate. By symmetry we assume that one of the rules corresponding to $i=1$ is applied. If Rule (13) is applied, then there exist locations $\mathfrak{l}, \mathfrak{l}^{\prime}, \mathfrak{l}^{\prime \prime}, \mathfrak{l}_{1}$ and $\mathfrak{l}_{2}$ such that $\mathfrak{h}(\mathfrak{l})=$ $\left(\mathfrak{l}^{\prime}, a, \mathfrak{l}_{1}, p_{1}, \mathfrak{l}_{2}, p_{2}\right)$ and $\mathfrak{h}\left(\mathfrak{l}_{1}\right)=\left(\mathfrak{l}^{\prime \prime}, n\right)$, where $a \in \mathbb{V}, n \in \llbracket 1, N \rrbracket, a \neq \mathbf{u}_{n}^{1}\left[p_{1}\right]$. Since $\mathfrak{h}$ is a PCP-encoding, this is possible only if $\mathfrak{l}=\ell_{l}$, for some $l \in \llbracket 1, L \rrbracket$ and $\mathfrak{l}_{1}^{\prime}=\ell_{g}^{\prime}$, for some $g \in \llbracket 1, G \rrbracket$. Then $\mathfrak{h}$ falsifies Cond. 1 of Def. 2 , hence $\mathfrak{h}$ is not 1 -consistent. If Rule (14) is applied then there exist locations $\mathfrak{l}, \mathfrak{l}^{\prime}, \mathfrak{l}^{\prime \prime}, \mathfrak{l}_{1}, \mathfrak{l}_{2}, \mathfrak{l}_{1}^{\prime}, \mathfrak{l}_{2}^{\prime}$ and $\mathfrak{l}_{1}^{\prime \prime}$ such that $\mathfrak{h}(\mathfrak{l})=\left(\mathfrak{l}^{\prime}, a, \mathfrak{l}_{1}, p_{1}, \mathfrak{l}_{2}, p_{2}\right), \mathfrak{h}\left(\mathfrak{l}^{\prime}\right)=\left(\mathfrak{l}^{\prime \prime}, a^{\prime}, \mathfrak{l}_{1}^{\prime}, p_{1}^{\prime}, \mathfrak{l}_{2}^{\prime}, p_{2}^{\prime}\right)$ and $\mathfrak{h}\left(\mathfrak{l}_{1}\right)=\left(\mathfrak{l}_{1}^{\prime \prime}, n\right)$, where $1 \leq p_{1}<\left|\mathbf{u}_{n}^{1}\right|$, and either $\mathfrak{l}_{1} \not \approx \mathfrak{l}_{1}^{\prime}$ or $p_{1}^{\prime} \neq p_{1}+1$. Since $\mathfrak{h}$ is a PCP-encoding, we must have $\mathfrak{l}=\ell_{l}$ and $\mathfrak{l}^{\prime}=\ell_{l+1}$ for some $l \in \llbracket 1, L \rrbracket$, and $\mathfrak{l}_{1}=\ell_{g}^{\prime}$, for some $g \in \llbracket 1, G \rrbracket$. Therefore, Cond. 2 of Def. 2 is falsified. The proof is similar if Rule (15) is applied: we have $p_{1}=\left|\mathbf{u}_{n}^{1}\right|$, and either $\mathfrak{l}_{1}^{\prime} \not \mathfrak{l}_{1}^{\prime \prime}$ or $p_{1}^{\prime} \neq 1$, which entails that Cond. 3 of Def. 2 is falsified.

$\Leftarrow$ : Assume that $\mathfrak{h}$ is not $i$-consistent, for some $i=1,2$. We assume that $i=1$, the proof for $i=2$ is symmetric. By definition of a PCP-encoding, there exists a store $\mathfrak{s}^{\prime}$ such that $\mathfrak{s}(x)=\mathfrak{s}^{\prime}(x)$ and:

$$
\left(\mathfrak{s}^{\prime}, \mathfrak{h}\right)=_{\mathcal{R}^{\pi}} *_{l=1}^{L} x_{l} \mapsto\left(x_{l+1}, a_{l}, y_{l}^{1}, p_{l}^{1}, y_{l}^{2}, p_{l}^{2}\right) * *_{g=1}^{G} y_{g} \mapsto\left(y_{g+1}, n_{g}\right),
$$

where $x_{l}, y_{l}^{i}, y_{g} \in \mathcal{V}, a_{l} \in \mathbb{V}, p_{l}^{i} \in \llbracket 1, M \rrbracket$ and $n_{g} \in \llbracket 1, N \rrbracket$, for all $l \in \llbracket 1, L \rrbracket, g \in$ $\llbracket 1, G \rrbracket$ and $i \in\{1,2\}$. The location $\mathfrak{s}\left(x_{1}\right)$ is the root of the heap, thus we have $\mathfrak{s}(x)=\mathfrak{s}\left(x_{1}\right)$, and we may assume, w.l.o.g., that $x=x_{1}$. Since by hypothesis $\mathfrak{h}$ is not 1 -consistent, there exist $l \in \llbracket 1, L \rrbracket$ and $g \in \llbracket 1, G \rrbracket$ such that one of the conditions in Def. 2 is falsified. Observe that, according to Def. 2, this entails that $\mathfrak{s}\left(y_{l}^{1}\right)=$ $\mathfrak{s}\left(y_{g}\right)$. We also have $l<L$; indeed, it is easy to check that the faulty location cannot be the last one, since, by definition of a PCP-encoding, this location is associated with the special character $\$$ and index $n_{G}=N$, with $\mathbf{u}_{N}^{i}=\$$ and $p_{L}^{i}=1$. We therefore have: $\left(\mathfrak{s}^{\prime}, \mathfrak{h}\right) \models_{\mathcal{R}^{\pi}} \phi_{1} * x_{l} \mapsto\left(x_{l+1}, a_{l}, y_{l}^{1}, p_{l}^{1}, y_{l}^{2}, p_{l}^{2}\right) * \phi_{2} * \psi_{1} * y_{g} \mapsto$ $\left(y_{g+1}, n_{g}\right) * \psi_{2}$, where:

$$
\begin{aligned}
& \phi_{1} \stackrel{\text { def }}{=} *_{l^{\prime}=1}^{l-1} x_{l^{\prime}} \mapsto\left(x_{l^{\prime}+1}, a_{l^{\prime}}, y_{l^{\prime}}^{1}, p_{l^{\prime}}^{1}, y_{l^{\prime}}^{2}, p_{l^{\prime}}^{2}\right) \quad \psi_{1} \stackrel{\text { def }}{=} *_{g^{\prime}=1}^{g-1} y_{g^{\prime}} \mapsto\left(y_{g^{\prime}+1}, n_{g^{\prime}}\right) \\
& \phi_{2} \stackrel{\text { def }}{=} *_{l^{\prime}=l+1}^{L} x_{l^{\prime}} \mapsto\left(x_{l^{\prime}+1}, a_{l^{\prime}}, y_{l^{\prime}}^{1}, p_{l^{\prime}}^{1}, y_{l^{\prime}}^{2}, p_{l^{\prime}}^{2}\right) \quad \psi_{2} \stackrel{\text { def }}{=} *_{g^{\prime}=g+1}^{G} y_{g^{\prime}} \mapsto\left(y_{g^{\prime}+1}, n_{g^{\prime}}\right)
\end{aligned}
$$


It is clear that $\phi_{2} \models_{\mathcal{R}^{\pi}} A\left(x_{l+1}\right)(\star)$ using Rule (7) $L-l$ times and Rule (8) once. Further, if $l<L-1$ then $\phi_{2} \models_{\mathcal{R}^{\pi}} x_{l+1} \mapsto\left(x_{l+2}, a_{l+1}, y_{1}^{l+1}, p_{1}^{l+1}, y_{2}^{l+1}, p_{2}^{l+1}\right) *$ $A\left(x_{l+2}\right)$. We also have $\phi_{2} \models_{\mathcal{R}^{\pi}} C\left(x_{l+1}, x_{l+2}, a_{l}+1, y_{1}^{l+1}, p_{1}^{l+1}, y_{2}^{l+1}, p_{2}^{l+1}\right)(\dagger)$, using Rule (9) if $l<L-1$ and Rule (10) otherwise. Similarly, $\psi_{2} \models_{\mathcal{R}^{\pi}} A\left(y_{g+1}\right)$ and $y_{g} \mapsto\left(y_{g+1}, n_{g}\right) * \psi_{2} \models_{\mathcal{R}^{\pi}} C\left(y_{g}, y_{g+1}, n_{g}, \perp, \perp, \perp, \perp\right)(\$)$. Furthermore, if $g>1$, then $\psi_{1}=\mathcal{R}^{\pi} A\left(y_{g}\right)$. Assume first that Cond. 1 is falsified. Then $a_{l} \neq \mathbf{u}_{g}^{1}\left[p_{l}^{1}\right]$, hence, by Rule (13), using $(\star)$ and $(\$)$, we get that $x_{l} \mapsto\left(x_{l+1}, a_{l}, y_{1}^{l}, p_{1}^{l}, y_{2}^{l}, p_{2}^{l}\right) *$ $\phi_{2} * y_{g} \mapsto\left(y_{g+1}, n_{g}\right) * \psi_{2} \models_{\mathcal{R}^{\pi}} B\left(x_{l}\right)$. If Cond. 2 or 3 are falsified, then we get the same result, using the rules (14) and (15), instead of (13), and Relation ( $\dagger$ ) instead of $(\star)$. If $g=1$, then, by applying Rule (12) $g-1$ times, we deduce that $(\mathfrak{s}, \mathfrak{h}) \models_{\mathcal{R}^{\pi}} B\left(x_{1}\right)$. If $g>1$, then we get the same result by applying Rule (11) once and Rule (12) $g-1$ times, using the fact that $\psi_{1} \models_{\mathcal{R}^{\pi}} A\left(y_{g}\right)$.

Putting everything together, we get the stated result:

Theorem 6. The entailment problem is undecidable for systems of inductive definitions satisfying the progress and connectivity condition, but not the establishment condition.

Proof. Consider an instance $\pi$ of the (adapted) PCP. By Lemma 3, the PCP has a solution iff there exists a PCP-encoding that is $i$-consistent, for $i=1,2$. By Lemmas 4 and 5 , we can compute a system of inductive rules $R^{\pi}$ such that $\mathfrak{h}$ is a PCP-encoding iff $(\mathfrak{s}, \mathfrak{h}) \models_{\mathfrak{R}^{\pi}} I\left(x_{1}\right)$, and $\mathfrak{h}$ is $i$-consistent for all $i=1,2$ iff $(\mathfrak{s}, \mathfrak{h}) \forall_{\mathfrak{R} \pi} B\left(x_{1}\right)$; i.e., such that the PCP has a solution iff $I\left(x_{1}\right) \forall_{\mathcal{R}^{\pi}} B\left(x_{1}\right)$. It is easy to check that $\mathcal{R}^{\pi}$ is progressing and connected but not established, see for instance Rules (3), (7), (8) and (12) in Fig. 2. Since the PCP is undecidable, the proof is completed.

\section{References}

[1] R. Iosif, A. Rogalewicz, J. Simacek, The tree width of separation logic with recursive definitions, in: Proc. of CADE-24, Vol. 7898 of LNCS, 2013.

[2] S. S. Ishtiaq, P. W. O'Hearn, Bi as an assertion language for mutable data structures, in: ACM SIGPLAN Notices, Vol. 36, 2001, pp. 14-26.

[3] B. Courcelle, The monadic second-order logic of graphs. I. Recognizable sets of finite graphs, Information and Computation 85 (1) (1990) 12 - 75. 
[4] J. Pagel, F. Zuleger, Beyond symbolic heaps: Deciding separation logic with inductive definitions, in: LPAR-23, Vol. 73 of EPiC Series in Computing, EasyChair, 2020, pp. 390-408.

[5] M. Echenim, R. Iosif, N. Peltier, Decidable entailments in separation logic with inductive definitions: Beyond establishment, in: CSL 2021: 29th International Conference on Computer Science Logic, EPiC Series in Computing, EasyChair, 2021. 